\title{
Genotypic Variation for High Temperature Tolerance in Relation to Carbon Partitioning and Grain Sink Activity in Wheat
}

\author{
Bavita Asthir $^{*}$, Puneet Kaur Rai ${ }^{1}$, Navtej S. Bains ${ }^{2}$, Virinder S. Sohu ${ }^{2}$ \\ ${ }^{1}$ Department of Biochemistry, Punjab Agricultural University, Ludhiana, India; ${ }^{2}$ Department of Plant Breeding and Genetics, Punjab \\ Agricultural University, Ludhiana, India. \\ Email: " b.asthir@rediffmail.com
}

Received September $22^{\text {nd }}, 2011$; revised October $25^{\text {th }}, 2011$; accepted November $23^{\text {rd }}, 2011$

\begin{abstract}
Effect of high temperature tolerance in relation to carbon partitioning and grain sink activity was studied in ten genotypes of wheat (viz C 306, PBW 343, PBW 502, PBW 509, PBW 521, PBW 522, PBW 550, Inqalab, WL 711 and Sonalika) under normal (November, $25.6^{\circ} \mathrm{C}$ during grain filling) and late planting conditions (December, $29.4^{\circ} \mathrm{C}$ ). Contents of reducing sugars first increased till actively metabolizing stage i.e. 14 days after anthesis (DAA) in parallel with the activity of acid invertase and thereafter declined. Significant reduction in total free sugars and sucrose content was observed during grain development. Acid invertase activity got induced especially in PBW 343 and C 306 under late sown conditions, whereas sucrose synthase, total amylase activities got repressed. Sucrose synthase was significantly high at all stages and it predominated over invertases. Neutral invertase and total amylase showed peak activity of one week earlier in the late sown crop as compared to normal sown crop. A premature cessation of starch deposition occurred in cvs PBW 521, PBW 522 under late sown conditions due to shortening of grain filling period. Our results suggest that decline in sugar content in spite of high sucrolytic enzymes may be correlated to more utilization of assimilates over production/translocation for grain sink activity under high temperature influences. Knowledge of the mechanisms that underlie carbohydrate partitioning in diverse genotypes could be exploited for generating germplasm that can tolerate high temperature.
\end{abstract}

Keywords: Carbohydrate Metabolism; Genotypes; Grains; Heat Stress; Sucrose; Starch; Triticum aestivum L.

\section{Introduction}

High temperature $\left(>30^{\circ} \mathrm{C}\right)$ at the time of grain filling is one of the major constraints in increasing productivity of wheat in tropical countries like India [1]. It has been reported that single grain mass falls by $3 \%-5 \%$ for every $1^{\circ} \mathrm{C}$ rise in temperature above $18^{\circ} \mathrm{C}$ [2]. About $50 \%$ of wheat area is planted after the optimum time and therefore, suffers heat stress which causes a significant yield loss. To sustain wheat productivity under late planting, research emphasis has been given to develop heat tolerant genotypes. Information on high temperature effects on various parameters associated with heat tolerance of wheat genotypes under field conditions are still inadequate [3]. A major perturbation of plant protection under heat stress is ascribed to carbon metabolism $[4,5]$ which is manifested in premature cessation of starch deposition in endosperm [6]. Supply of assimilates is never the limiting factor rather its utilization within the grain is the

\footnotetext{
${ }^{*}$ Corresponding author.
}

controlling factor of sink activity $[7,8]$.

Sucrose upon entering into the grain is metabolized by invertases, sucrose synthases and sucrose-phosphate synthase $[9,10]$. Activity of these enzymes has been associated with the developmental processes of sinks [11-13] and they appear to be potential targets of regulation in improving grain filling processes and yield in wheat under heat stress [14]. Improving the genetic adaptations of wheat cultivars to heat stress is an important objective in the breeding programs. Some genotypes are reported to have a thermotolerant response and could be used as genetic sources for heat tolerance [15].

Grain filling in cereals is mainly determined by sink strength $[16,17]$. The sink strength can be described as the product of sink size and sink activity [16]. Sink activeity is a physiological restraint that includes multiple fators and key enzymes involved in carbohydrate utilizetion and storage. Enhanced sink activity under water deficient may be attributed to increased carbon remobilizetion from the stems to grains or accelerated grain filling 
[10]. Since starch is mainly synthesized from sucrose, which is the major translocatory form of carbon in the phloem from source to sink tissues, therefore its catabolism leads to carbon formation for starch synthesis. Reports in the literature indicate that supply of photoassimilate occur mainly in the form of sucrose in sink organs like seed, which has to be cleaved and resynthesized [18]. In wheat, sucrose enters as such without prior hydrolysis in the endosperm [19]. Following entry into the grain, sucrolytic enzymes are adversely affected under high temperature stress in rice [13], but there have been no such reports in wheat on genotypic variation for high temperature tolerance. Since, late sown crop experiences heat stress during grain filling period as compared to normal sown crop, therefore, studying grain sink activity and carbon partitioning under these conditions will help the breeders to raise heat tolerant crops.

\section{Materials and Methods}

\subsection{Plant Material and Growth Conditions}

Wheat (Triticum aestivum L.) genotypes namely C 306 , PBW 343, PBW 502, PBW 509, PBW 521, PBW 522, PBW 550, Inqalab, WL 711 and Sonalika were procured from the Department of Plant Breeding and Genetics, Punjab Agricultural University, Ludhiana. Genotypes were tested for differential response to high temperature tolerance at 7, 14, 21, 28 and 40 days after anthesis (DAA) for carbon partitioning and enzymic studies. Fresh tissue was used for enzyme assays whereas stored tissue was used for sugar analysis. These genotypes were sown on 12th November 2006 (normal sown) and also on $15^{\text {th }}$ December 2006 (late sown) in order to expose the plants to different temperature regimes. Normal recommended agronomic practices were performed. The temperatures under two sowings at three stages were recorded by a field meterological laboratory fitted with microprocessor controlled data logger, which recorded daily maximum/ minimum temperature. The mean temperatures under normal sown crop at vegetative, anthesis and post anthesis stages were $11.8^{\circ} \mathrm{C}, 14.5^{\circ} \mathrm{C}$ and $25.6^{\circ} \mathrm{C}$ whereas in the late sown crop mean temperatures were $13.0^{\circ} \mathrm{C}, 19.8^{\circ} \mathrm{C}$ and $29.4^{\circ} \mathrm{C}$, respectively. Anthesis appeared approximately between 85 to 90 days after planting and grain filling duration was completed in 40 DAA.

\subsection{Extraction and Estimation of Free Sugars, Starch and Protein}

Free sugars were extracted sequentially with $80 \%$ and $70 \%$ ethanol. The extracts containing sugars were concentrated by evaporating off the ethanol under vacuum. Re- ducing sugars and sucrose were determined colorimetrically [20]. From the sugar free residue so obtained, starch was extracted with cold $\left(2^{\circ} \mathrm{C}-4^{\circ} \mathrm{C}\right)$ perchloric $(52 \%)$, purified with iodine precipitation and estimated as described above. Soluble proteins were extracted in $0.1 \mathrm{M}$ $\mathrm{NaOH}$ and precipitated with trichloroacetic acid [21].

\subsection{Extraction and Assay of Enzymes}

From freshly collected samples, soluble acid invertase (EC 3.2.1.26; pH 4.8), soluble neutral invertase (EC 3.2.1.27; $\mathrm{pH} 7.5$ ) and sucrose synthase (synthesis, EC 2.4.1.13), were extracted by the procedure employed by Asthir et al. [20]. Grain samples (4 g) were homogenized at $0^{\circ} \mathrm{C}-4^{\circ} \mathrm{C}$ in $50 \mathrm{mM}$ Hepes-NaOH buffer (pH 7.5) containing $5 \mathrm{mM} \mathrm{MgCl} 2,1 \mathrm{mM}$ sodium EDTA, $2.5 \mathrm{mM}$ DTT, $0.5 \mathrm{mg} \cdot \mathrm{ml}^{-1}$ BSA and $0.05 \%(\mathrm{v} / \mathrm{v})$ Triton X100. Homogenates were centrifuged at $10,000 \mathrm{~g}$ for $15 \mathrm{~min}$ and the pellets resuspended in extraction buffer and centrifuged. The supernatants were pooled and passed through Sephadex G-25 column $(17 \mathrm{~cm})$ equilibrated with the above buffer without EDTA and Triton X-100.

Soluble acid and soluble neutral invertases were assayed from the test extracts [20]. The reaction mixture (1 $\mathrm{ml}$ ) consisted of $0.6 \mathrm{ml}$ of $0.2 \mathrm{M} \mathrm{Na}$ acetate buffer $\mathrm{pH} 4.8$ (for soluble acid invertase) or $0.2 \mathrm{M} \mathrm{Na}$ phosphate, $\mathrm{pH}$ 7.5 (for soluble neutral invertase), $0.2 \mathrm{ml}$ of $250 \mathrm{mM}$ sucrose and $0.2 \mathrm{ml}$ of enzyme extract. The contents were incubated at $37^{\circ} \mathrm{C}$ for $20 \mathrm{~min}$ and the reaction was terminated by addition of $1 \mathrm{ml}$ of Nelson reagent $\mathrm{C}$. The amount of reducing sugars were measured [22] and the concentration of hydrolysed sucrose was calculated by multiplying the reducing sugar concentration by the factor of 0.95 . Sucrose synthase was assayed from the test extracts as described [20]. The reaction mixture $(0.5 \mathrm{ml})$ for sucrose synthase (synthesis) contained $3 \mathrm{mM}$ UDPG, $10 \mathrm{mM}$ fructose, $5 \mathrm{mM} \mathrm{MgSO}_{4}, 40 \mathrm{mM}$ Tris- $\mathrm{HCl}$ buffer $(\mathrm{pH} 8.2)$ and $0.2 \mathrm{ml}$ enzyme preparation. After incubation at $37^{\circ} \mathrm{C}$ for $30 \mathrm{~min}, 0.1 \mathrm{ml}$ of $30 \% \mathrm{KOH}$ was added. The contents were heated for $20 \mathrm{~min}$ in boiling water bath to destroy free fructose. One $\mathrm{ml}$ of resorcinol $(0.15$ glacial acetic acid $+0.25 \mathrm{~g}$ thiourea) and $3 \mathrm{ml}$ of $30 \%$ $\mathrm{HCl}$ were added. The contents were mixed thoroughly and incubated at $80^{\circ} \mathrm{C}$ for $10 \mathrm{~min}$ and absorbance was read at $490 \mathrm{~nm}$.

\subsection{Statistical Analysis}

Experiment was laid out in complete randomized block design with three replications. Data was analysed for analysis of variance by factorial randomized design and least significant differences were calculated. In tables \pm values represented standard error of the means. 


\section{Results}

During vegetative, anthesis and post anthesis stages under normal and late sown conditions, genotypes experienced temperature difference of $1.2^{\circ} \mathrm{C}, 5.3^{\circ} \mathrm{C}$ and $3.8^{\circ} \mathrm{C}$, respectively. A reduction of 4 percent in grain weight for each $1{ }^{\circ} \mathrm{C}$ increase in mean air temperature during grainfilling was reported earlier [23].

\subsection{Effect of High Temperature on Free Sugar Content}

Reducing sugar (RS) and sucrose contents declined throughout grain development from 7 to 40 DAA in all genotypes (Tables 1 and 2). Under normal sown conditions PBW 343 had the highest content of total sugar (RS and sucrose) followed by $\mathrm{C} 306$. The genotypes Inqalab and PBW 521 had the least levels of total sugars content.
Whereas the contents of RS first increased till 14 DAA and then declined continuously till maturity (Table 1) under normal and late sown conditions. Reducing sugar content was comparatively high in the genotypes PBW 343 (9.1 mg/g DW) and PBW 550 (7.7 mg/g DW) and it was lowest in PBW 521 (4.1 mg/g DW) and WL 711 (5.1 $\mathrm{mg} / \mathrm{g} \mathrm{DW}$ ) at 7 DAA under normal sown conditions. However, the increased content of RS in these genotypes at 14 DAA can be correlated with peak activity of acid invertase which was maximum at 14 DAA (Table 4).

Sucrose predominated over reducing sugars in all the genotypes at all stages of grain development both under normal and late sown conditions (Table 2). The genotypes PBW 343 and C 306 had the highest levels of sucrose content at 7 DAA, respectively. Genotypes containing the least levels of sucrose were PBW 521 and Inqalab.

Table 1. Level of reducing sugars (mg/g DW) in developing grains of different genotypes of wheat under normal and late sown conditions.

\begin{tabular}{|c|c|c|c|c|c|c|}
\hline \multirow{2}{*}{\multicolumn{2}{|c|}{ Genotypes Normal/Late sown }} & \multicolumn{5}{|c|}{ Reducing sugars } \\
\hline & & \multicolumn{5}{|c|}{ Days after anthesis (DAA) } \\
\hline & & 7 & 14 & 21 & 28 & 40 \\
\hline \multirow{2}{*}{ С 306} & NS & $7.4 \pm 0.56$ & $8.5 \pm 0.33$ & $3.7 \pm 0.24$ & $2.2 \pm 0.13$ & $2.0 \pm 0.12$ \\
\hline & LS & $6.5 \pm 0.38$ & $6.7 \pm 0.21$ & $2.6 \pm 0.11$ & $1.8 \pm 0.09$ & $1.4 \pm 0.09$ \\
\hline \multirow{2}{*}{ PBW 343} & NS & $9.1 \pm 0.42$ & $10.7 \pm 2.66$ & $5.6 \pm 0.39$ & $2.6 \pm 0.19$ & $1.8 \pm 0.10$ \\
\hline & $\mathbf{L S}$ & $7.5 \pm 0.33$ & $5.8 \pm 0.29$ & $3.3 \pm 0.21$ & $1.8 \pm 0.08$ & $0.9 \pm 0.05$ \\
\hline \multirow{2}{*}{ PBW 502} & NS & $7.6 \pm 0.44$ & $7.8 \pm 0.26$ & $3.4 \pm 0.27$ & $2.0 \pm 0.12$ & $0.8 \pm 0.05$ \\
\hline & LS & $4.3 \pm 0.27$ & $6.2 \pm 0.13$ & $2.5 \pm 0.18$ & $1.2 \pm 0.07$ & $0.3 \pm 0.02$ \\
\hline \multirow{2}{*}{ PBW 509} & NS & $6.1 \pm 0.34$ & $8.5 \pm 0.52$ & $5.2 \pm 0.39$ & $2.0 \pm 0.04$ & $1.0 \pm 0.05$ \\
\hline & LS & $5.2 \pm 0.28$ & $6.5 \pm 0.50$ & $3.8 \pm 0.26$ & $1.9 \pm 0.11$ & $0.4 \pm 0.02$ \\
\hline \multirow{2}{*}{ PBW 521} & NS & $4.1 \pm 0.20$ & $5.4 \pm 0.37$ & $4.4 \pm 0.33$ & $3.7 \pm 0.17$ & $1.7 \pm 0.09$ \\
\hline & LS & $3.2 \pm 0.16$ & $4.5 \pm 0.39$ & $3.0 \pm 0.18$ & $2.4 \pm 0.12$ & $0.8 \pm 0.05$ \\
\hline \multirow{2}{*}{ PBW 522} & NS & $7.1 \pm 0.43$ & $9.2 \pm 0.74$ & $6.0 \pm 0.41$ & $2.8 \pm 0.16$ & $1.2 \pm 0.08$ \\
\hline & LS & $5.6 \pm 0.26$ & $6.5 \pm 0.55$ & $4.3 \pm 0.30$ & $2.1 \pm 0.11$ & $0.8 \pm 0.06$ \\
\hline \multirow{2}{*}{ PBW 550} & NS & $7.7 \pm 0.52$ & $10.7 \pm 0.81$ & $6.5 \pm 0.39$ & $3.2 \pm 0.25$ & $2.0 \pm 0.09$ \\
\hline & LS & $6.0 \pm 0.40$ & $8.0 \pm 0.62$ & $5.1 \pm 0.26$ & $3.0 \pm 0.13$ & $1.3 \pm 0.08$ \\
\hline \multirow{2}{*}{ Inqalab } & NS & $6.5 \pm 0.47$ & $7.7 \pm 0.36$ & $3.0 \pm 0.12$ & $2.0 \pm 0.11$ & $1.2 \pm 0.09$ \\
\hline & LS & $5.3 \pm 0.33$ & $6.5 \pm 0.41$ & $2.5 \pm 0.19$ & $1.6 \pm 0.07$ & $0.9 \pm 0.05$ \\
\hline \multirow{2}{*}{ WL 711} & NS & $5.1 \pm 0.34$ & $6.3 \pm 0.38$ & $4.2 \pm 0.22$ & $2.5 \pm 0.13$ & $1.6 \pm 0.10$ \\
\hline & LS & $4.2 \pm 0.27$ & $5.1 \pm 0.27$ & $3.7 \pm 0.15$ & $3.8 \pm 0.21$ & $0.8 \pm 0.04$ \\
\hline \multirow{2}{*}{ Sonalika } & NS & $7.4 \pm 0.67$ & $8.1 \pm 0.90$ & $7.3 \pm 0.40$ & $3.0 \pm 0.17$ & $1.0 \pm 0.05$ \\
\hline & LS & $6.4 \pm 0.46$ & $8.3 \pm 0.71$ & $4.8 \pm 0.21$ & $2.2 \pm 0.10$ & $0.4 \pm 0.02$ \\
\hline
\end{tabular}


Table 2. Level of sucrose (mg/g DW) in developing grains of different genotypes of wheat under normal and late sown conditions.

\begin{tabular}{|c|c|c|c|c|c|c|}
\hline \multirow{2}{*}{\multicolumn{2}{|c|}{ Genotypes normal/late sown }} & \multicolumn{5}{|c|}{ Sucrose } \\
\hline & & \multicolumn{5}{|c|}{ Days after anthesis (DAA) } \\
\hline & & 7 & 14 & 21 & 28 & 40 \\
\hline \multirow{2}{*}{ C 306} & NS & $112 \pm 7.65$ & $74 \pm 5.49$ & $45 \pm 1.00$ & $16 \pm 0.48$ & $5 \pm 0.37$ \\
\hline & $\mathbf{L S}$ & $80 \pm 6.49$ & $54 \pm 3.18$ & $30 \pm 0.85$ & $11 \pm 0.34$ & $2 \pm 0.18$ \\
\hline \multirow{2}{*}{ PBW 343} & NS & $133 \pm 8.48$ & $70 \pm 4.65$ & $39 \pm 0.84$ & $16 \pm 0.66$ & $4 \pm 0.28$ \\
\hline & $\mathbf{L S}$ & $97 \pm 5.24$ & $61 \pm 2.42$ & $31 \pm 0.72$ & $9 \pm 0.45$ & $3 \pm 0.16$ \\
\hline \multirow{2}{*}{ PBW 502} & NS & $93 \pm 5.67$ & $66 \pm 4.68$ & $48 \pm 2.15$ & $16 \pm 0.70$ & $4 \pm 0.21$ \\
\hline & LS & $81 \pm 3.53$ & $53 \pm 3.47$ & $37 \pm 1.19$ & $9 \pm 0.44$ & $2 \pm 0.10$ \\
\hline \multirow{2}{*}{ PBW 509} & NS & $96 \pm 6.46$ & $61 \pm 4.65$ & $32 \pm 1.01$ & $13 \pm 0.68$ & $5 \pm 0.35$ \\
\hline & $\mathbf{L S}$ & $74 \pm 4.12$ & $49 \pm 2.71$ & $16 \pm 0.78$ & $10 \pm 0.79$ & $2 \pm 0.19$ \\
\hline \multirow{2}{*}{ PBW 521} & NS & $78 \pm 4.63$ & $66 \pm 4.82$ & $30 \pm 2.23$ & $14 \pm 0.31$ & $4 \pm 0.13$ \\
\hline & $\mathbf{L S}$ & $65 \pm 3.27$ & $42 \pm 1.69$ & $21 \pm 1.02$ & $6 \pm 0.48$ & $3 \pm 0.09$ \\
\hline \multirow{2}{*}{ PBW 522} & NS & $92 \pm 6.62$ & $70 \pm 3.49$ & $56 \pm 2.52$ & $15 \pm 0.21$ & $3 \pm 0.09$ \\
\hline & LS & $77 \pm 4.89$ & $58 \pm 1.62$ & $36 \pm 1.84$ & $8 \pm 0.19$ & $2 \pm 0.04$ \\
\hline \multirow{2}{*}{ PBW 550} & NS & $91 \pm 6.54$ & $63 \pm 4.57$ & $29 \pm 1.62$ & $12 \pm 0.09$ & $5 \pm 0.27$ \\
\hline & LS & $64 \pm 4.59$ & $46 \pm 2.63$ & $18 \pm 1.04$ & $6 \pm 0.06$ & $2 \pm 0.10$ \\
\hline \multirow{2}{*}{ Inqalab } & NS & $71 \pm 3.87$ & $58 \pm 2.89$ & $49 \pm 2.09$ & $22 \pm 1.23$ & $4 \pm 0.16$ \\
\hline & LS & $54 \pm 2.91$ & $38 \pm 2.64$ & $34 \pm 1.63$ & $17 \pm 1.05$ & $2 \pm 0.09$ \\
\hline \multirow{2}{*}{ WL 711} & NS & $106 \pm 8.87$ & $60 \pm 3.13$ & $29 \pm 1.47$ & $9 \pm 0.73$ & $4 \pm 0.26$ \\
\hline & LS & $90 \pm 4.45$ & $48 \pm 2.93$ & $19 \pm 0.86$ & $6 \pm 0.42$ & $2 \pm 0.08$ \\
\hline \multirow{2}{*}{ Sonalika } & NS & $110 \pm 4.32$ & $79 \pm 4.69$ & $27 \pm 1.22$ & $12 \pm 0.61$ & $4 \pm 0.19$ \\
\hline & LS & $86 \pm 3.31$ & $46 \pm 2.72$ & $21 \pm 1.25$ & $9 \pm 0.43$ & $3 \pm 0.12$ \\
\hline
\end{tabular}

\subsection{Effect of High Temperature on Carbohydrate Metabolism}

Changes in sucrose to starch metabolizing enzymes viz. invertase, sucrose synthase and total amylase in ten genotypes of wheat under normal and late sown conditions were recorded. Sucrose synthase (synthesis direction) showed peak activity at 7 DAA under normal and late sown conditions, followed by continuous decline till grain maturity (Table 3). Heat stress experienced by genotypes under late sown conditions caused reduction in the activity of sucrose synthase.

Total activity of acid invertase in ten genotypes of wheat at different stages of grain development is shown in Table 4. The activity of acid invertase increased till 14 DAA and then decreased till maturity. At 14 DAA under normal sown conditions, the activity of acid invertase was significantly high in C 306 (182 $\mu$ g sucrose hydro- lyzed/min/g FW) and PBW 343 (175 $\mu \mathrm{g}$ sucrose hydrolyzed/ $\mathrm{min} / \mathrm{g} \mathrm{FW}$ ). Whereas under late sown conditions the activity of acid invertase significantly increased in cv PBW $502(282 \mu \mathrm{g}$ sucrose hydrolyzed/min/g FW) followed by PBW $343(280 \mu \mathrm{g}$ sucrose hydrolyzed $/ \mathrm{min} / \mathrm{g}$ FW). C 306, WL 711 and PBW 509 also had higher values of acid invertase than other genotypes at 14 DAA. The activity of acid invertase in susceptible genotypes was more at initial and at later stages of the grain development i.e. at 7 and 40 DAA (PBW 509, PBW 521, PBW 522, PBW 550, Sonalika) whereas at all other stages of grain development activity of acid invertase in tolerant genotypes (C 306, PBW 343, PBW 502, WL 711 and Inqalab) was more. Activity of acid invertase at 7, 14 and 28 DAA was more in late sown than in early sown genotypes.

PBW 343 had highest activity of neutral invertase, 
Table 3. Total activity of sucrose synthase ( $\mu \mathrm{g}$ sucrose formed/min/g FW) in the developing grains of wheat under normal and late sown conditions.

\begin{tabular}{|c|c|c|c|c|c|c|}
\hline \multirow{2}{*}{\multicolumn{2}{|c|}{ Genotypes normal/late sown }} & \multicolumn{5}{|c|}{ Sucrose synthase } \\
\hline & & \multicolumn{5}{|c|}{ Days after anthesis (DAA) } \\
\hline & & 7 & 14 & 21 & 28 & 40 \\
\hline \multirow{2}{*}{ C 306} & NS & $320 \pm 21.74$ & $283 \pm 27.61$ & $221 \pm 21.73$ & $160 \pm 10.25$ & $80 \pm 6.48$ \\
\hline & LS & $287 \pm 16.89$ & $241 \pm 8.38$ & $142 \pm 11.92$ & $126 \pm 9.47$ & $64 \pm 5.03$ \\
\hline \multirow{2}{*}{ PBW 343} & NS & $284 \pm 16.36$ & $291 \pm 20.80$ & $220 \pm 16.41$ & $158 \pm 10.43$ & $72 \pm 4.34$ \\
\hline & $\mathbf{L S}$ & $262 \pm 22.47$ & $256 \pm 17.13$ & $140 \pm 11.33$ & $127 \pm 9.69$ & $60 \pm 4.12$ \\
\hline \multirow{2}{*}{ PBW 502} & NS & $266 \pm 20.81$ & $243 \pm 21.46$ & $147 \pm 10.18$ & $136 \pm 10.58$ & $71 \pm 5.73$ \\
\hline & $\mathbf{L S}$ & $220 \pm 17.46$ & $205 \pm 16.27$ & $140 \pm 9.78$ & $114 \pm 8.75$ & $55 \pm 3.71$ \\
\hline \multirow{2}{*}{ PBW 509} & NS & $238 \pm 18.29$ & $207 \pm 19.61$ & $162 \pm 10.72$ & $105 \pm 8.44$ & $59 \pm 2.14$ \\
\hline & LS & $180 \pm 14.66$ & $151 \pm 13.70$ & $101 \pm 8.75$ & $85 \pm 5.29$ & $44 \pm 3.71$ \\
\hline \multirow{2}{*}{ PBW 521} & NS & $229 \pm 11.20$ & $207 \pm 14.28$ & $168 \pm 12.38$ & $123 \pm 9.84$ & $45 \pm 2.84$ \\
\hline & LS & $185 \pm 16.38$ & $155 \pm 10.67$ & $86 \pm 7.23$ & $75 \pm 7.89$ & $36 \pm 2.12$ \\
\hline \multirow{2}{*}{ PBW 522} & NS & $222 \pm 17.64$ & $220 \pm 20.86$ & $178 \pm 13.61$ & $118 \pm 10.78$ & $51 \pm 1.67$ \\
\hline & LS & $189 \pm 11.99$ & $176 \pm 12.49$ & $93 \pm 5.18$ & $82 \pm 4.32$ & $40 \pm 2.79$ \\
\hline \multirow{2}{*}{ PBW 550} & NS & $234 \pm 19.81$ & $229 \pm 18.65$ & $193 \pm 13.61$ & $102 \pm 7.63$ & $55 \pm 2.65$ \\
\hline & LS & $197 \pm 13.31$ & $171 \pm 10.62$ & $91 \pm 5.39$ & $85 \pm 4.87$ & $47 \pm 3.52$ \\
\hline \multirow{2}{*}{ Inqalab } & NS & $279 \pm 10.56$ & $251 \pm 14.77$ & $133 \pm 10.22$ & $130 \pm 9.55$ & $69 \pm 4.61$ \\
\hline & $\mathbf{L S}$ & $205 \pm 17.43$ & $191 \pm 8.71$ & $106 \pm 8.42$ & $87 \pm 6.47$ & $55 \pm 3.42$ \\
\hline \multirow{2}{*}{ WL 711} & NS & $267 \pm 12.73$ & $240 \pm 20.58$ & $129 \pm 8.48$ & $121 \pm 10.61$ & $74 \pm 5.85$ \\
\hline & LS & $201 \pm 14.18$ & $180 \pm 12.43$ & $129 \pm 10.67$ & $106 \pm 9.22$ & $87 \pm 5.85$ \\
\hline \multirow{2}{*}{ Sonalika } & NS & $261 \pm 23.70$ & $219 \pm 18.62$ & $146 \pm 10.43$ & $141 \pm 10.68$ & $62 \pm 5.42$ \\
\hline & LS & $209 \pm 18.22$ & $170 \pm 11.47$ & $92 \pm 4.16$ & $80 \pm 5.14$ & $46 \pm 2.63$ \\
\hline
\end{tabular}

whereas PBW 521 had the lowest activity at 14 and 21 DAA under normal and late sown conditions (Table 5) which might account for high and low content of reducing sugars in PBW 343 and PBW 521, respectively.

The activities of total amylase during grain development are shown in Table 6. Under normal sown conditions, the total amylase activity increased till 28 DAA but under late sown conditions peak activity was observed at 21 DAA followed by a continuous decline. Genotypes under late sown conditions had lower values of total amylase activity. PBW 343 had the highest amylase activity whereas PBW 521 had the lowest amylase activity at 21 DAA.

\section{Discussion}

Irrespective of normal and late sown conditions, there was continuous decline of total sugars ( $\mathrm{RS}+$ sucrose) in all the genotypes which may be correlated to their rapid utilization for the synthesis of carbohydrate polymer mainly starch. In late sown genotypes there was more decline in total sugar content. Since, temperature was also high during late sown condition, so it is quite likely that higher utilization of sugars prevails during stress conditions. It has also been reported that free sugars decline during biotic or abiotic stresses to overcome stress and sugars are essential to plant growth and metabolism both as energy source and structural components [24]. A higher concentration of sucrose was observed in hybrid corn seeds tolerant to high drying temperatures [25]. The sucrose entering the grain either as such or after inversion is utilized for energy purpose, starch and cell wall polysaccharide synthesis and for the formation of other cellular products. 
Table 4. Total activity of acid invertase ( $\mu \mathrm{g}$ sucrose hydrolyzed/min/g FW) in developing grains of different genotypes of wheat under normal and late sown conditions.

\begin{tabular}{|c|c|c|c|c|c|c|}
\hline \multirow{2}{*}{\multicolumn{2}{|c|}{ Genotypes normal/late sown }} & \multicolumn{5}{|c|}{ Acid invertase } \\
\hline & & \multicolumn{5}{|c|}{ Days after anthesis (DAA) } \\
\hline & & 7 & 14 & 21 & 28 & 40 \\
\hline \multirow{2}{*}{ C 306} & NS & $114 \pm 9.65$ & $182 \pm 8.64$ & $161 \pm 12.79$ & $80 \pm 6.34$ & $61 \pm 4.68$ \\
\hline & $\mathbf{L S}$ & $117 \pm 8.64$ & $261 \pm 17.83$ & $107 \pm 8.48$ & $95 \pm 5.52$ & $54 \pm 2.53$ \\
\hline \multirow{2}{*}{ PBW 343} & NS & $114 \pm 8.21$ & $175 \pm 9.49$ & $155 \pm 10.68$ & $70 \pm 5.29$ & $53 \pm 3.67$ \\
\hline & $\mathbf{L S}$ & $125 \pm 9.78$ & $280 \pm 17.47$ & $95 \pm 8.41$ & $90 \pm 4.43$ & $59 \pm 4.12$ \\
\hline \multirow{2}{*}{ PBW 502} & NS & $105 \pm 7.43$ & $151 \pm 8.68$ & $137 \pm 7.55$ & $68 \pm 3.46$ & $47 \pm 2.78$ \\
\hline & LS & $125 \pm 6.49$ & $282 \pm 20.61$ & $106 \pm 8.76$ & $79 \pm 4.73$ & $66 \pm 4.07$ \\
\hline \multirow{2}{*}{ PBW 509} & NS & $168 \pm 11.85$ & $178 \pm 13.48$ & $81 \pm 3.88$ & $77 \pm 4.49$ & $61 \pm 4.63$ \\
\hline & $\mathbf{L S}$ & $104 \pm 7.36$ & $247 \pm 20.86$ & $62 \pm 5.81$ & $81 \pm 6.16$ & $82 \pm 5.89$ \\
\hline \multirow{2}{*}{ PBW 521} & NS & $151 \pm 9.45$ & $146 \pm 8.15$ & $110 \pm 6.42$ & $85 \pm 5.02$ & $72 \pm 6.18$ \\
\hline & LS & $166 \pm 7.35$ & $218 \pm 19.57$ & $72 \pm 6.07$ & $89 \pm 6.72$ & $87 \pm 7.42$ \\
\hline \multirow{2}{*}{ PBW 522} & NS & $158 \pm 5.42$ & $139 \pm 10.78$ & $125 \pm 9.61$ & $77 \pm 5.94$ & $70 \pm 5.63$ \\
\hline & LS & $150 \pm 6.78$ & $213 \pm 19.68$ & $53 \pm 2.58$ & $82 \pm 4.82$ & $76 \pm 6.49$ \\
\hline \multirow{2}{*}{ PBW 550} & NS & $133 \pm 9.68$ & $186 \pm 11.90$ & $108 \pm 8.47$ & $64 \pm 3.92$ & $61 \pm 3.69$ \\
\hline & LS & $130 \pm 11.52$ & $212 \pm 13.52$ & $47 \pm 2.69$ & $78 \pm 3.47$ & $74 \pm 5.41$ \\
\hline \multirow{2}{*}{ Inqalab } & NS & $113 \pm 8.67$ & $155 \pm 10.53$ & $123 \pm 7.95$ & $76 \pm 5.47$ & $56 \pm 3.56$ \\
\hline & LS & $113 \pm 9.48$ & $205 \pm 15.65$ & $84 \pm 4.52$ & $84 \pm 6.61$ & $60 \pm 2.42$ \\
\hline \multirow{2}{*}{ WL 711} & NS & $105 \pm 7.09$ & $147 \pm 10.31$ & $125 \pm 8.67$ & $68 \pm 4.39$ & $59 \pm 2.16$ \\
\hline & LS & $111 \pm 8.28$ & $231 \pm 21.42$ & $65 \pm 4.62$ & $77 \pm 5.71$ & $66 \pm 3.68$ \\
\hline \multirow{2}{*}{ Sonalika } & NS & $164 \pm 12.71$ & $163 \pm 13.38$ & $121 \pm 9.63$ & $72 \pm 4.17$ & $55 \pm 3.87$ \\
\hline & LS & $156 \pm 11.42$ & $169 \pm 12.52$ & $65 \pm 4.31$ & $71 \pm 3.35$ & $63 \pm 4.52$ \\
\hline
\end{tabular}

Sucrose synthase activity per unit fresh weight basis in endosperm decreased significantly as wheat kernel matured [26]. The activity of sucrose synthase was maximum in the genotypes PBW 343 and C 306. This corresponded to the maximum level of sucrose in these genotypes at both normal and late sown conditions. It is well accepted that sucrose metabolism is involved in responses to environmental stresses in many plant species [27]. Reports in the literature indicate that the composition of protein and starch in wheat grain could be easily manipulated by varying concentration of sucrose and glutamine and their ratio in the culture medium [28]. Sucrose and its non reducing derivatives offer several advantages to the plant. Besides their high water solubility, these sugars are readily synthesized from early products of photosynthesis or from the remobilization of storage products such as starch, structural carbohydrates and lipids.

Sucrose synthase activity predominated over invertases during grain development [29]. Pathway of sucrose conversion may depend on the processes occurring in the tissue at that time. Thus in the actively growing and storing sink tissues, sucrose synthase activity predominates and in others particularly those undergoing expansion acid invertase predominates. The concept of two distinct pathways for sucrose degradation was reported earlier [30], one mediated by sucrose synthase and other by invertases. Sucrose synthase another sucrose cleaving enzyme in addition to invertase also plays an important role as sucrose cleaving enzyme in carbohydrate metabolism, which in presence of UDP converts sucrose into UDP-glucose and fructose [31]. The UDP glucose provides a substrate that can be used directly or after modification and transported to other cellular components for 
Table 5. Total activity of alkaline invertase ( $\mu \mathrm{g}$ sucrose hydrolyzed/ $\mathrm{min} / \mathrm{g} \mathrm{FW}$ ) in the developing grains of different genotypes of wheat under normal and late sown conditions.

\begin{tabular}{|c|c|c|c|c|c|c|}
\hline \multirow{2}{*}{\multicolumn{2}{|c|}{ Genotypes normal/late sown }} & \multicolumn{5}{|c|}{ Alkaline invertase } \\
\hline & & \multicolumn{5}{|c|}{ Days after anthesis (DAA) } \\
\hline & & 7 & 14 & 21 & 28 & 40 \\
\hline \multirow{2}{*}{ C 306} & NS & $30 \pm 2.08$ & $48 \pm 2.72$ & $114 \pm 9.50$ & $57 \pm 2.49$ & $27 \pm 1.13$ \\
\hline & $\mathbf{L S}$ & $42 \pm 3.16$ & $107 \pm 8.84$ & $85 \pm 6.47$ & $57 \pm 3.72$ & $43 \pm 2.48$ \\
\hline \multirow{2}{*}{ PBW 343} & NS & $26 \pm 1.67$ & $50 \pm 2.45$ & $120 \pm 8.72$ & $59 \pm 2.87$ & $29 \pm 1.43$ \\
\hline & $\mathbf{L S}$ & $42 \pm 2.48$ & $113 \pm 9.29$ & $90 \pm 6.09$ & $67 \pm 4.61$ & $42 \pm 2.80$ \\
\hline \multirow{2}{*}{ PBW 502} & NS & $29 \pm 1.35$ & $38 \pm 2.23$ & $65 \pm 3.66$ & $52 \pm 3.12$ & $26 \pm 1.76$ \\
\hline & $\mathbf{L S}$ & $35 \pm 1.66$ & $103 \pm 7.57$ & $83 \pm 6.48$ & $65 \pm 2.58$ & $38 \pm 2.81$ \\
\hline \multirow{2}{*}{ PBW 509} & NS & $30 \pm 1.20$ & $37 \pm 1.25$ & $110 \pm 8.65$ & $43 \pm 2.48$ & $37 \pm 2.26$ \\
\hline & $\mathbf{L S}$ & $34 \pm 2.38$ & $80 \pm 4.36$ & $42 \pm 2.43$ & $30 \pm 2.63$ & $42 \pm 2.81$ \\
\hline \multirow{2}{*}{ PBW 521} & NS & $38 \pm 2.42$ & $47 \pm 2.13$ & $49 \pm 2.30$ & $44 \pm 1.69$ & $40 \pm 2.41$ \\
\hline & $\mathbf{L S}$ & $40 \pm 2.81$ & $76 \pm 5.41$ & $34 \pm 1.42$ & $34 \pm 1.37$ & $46 \pm 3.17$ \\
\hline \multirow{2}{*}{ PBW 522} & NS & $41 \pm 3.64$ & $40 \pm 3.42$ & $56 \pm 2.35$ & $40 \pm 2.38$ & $41 \pm 2.68$ \\
\hline & $\mathbf{L S}$ & $45 \pm 2.85$ & $79 \pm 6.29$ & $45 \pm 2.64$ & $29 \pm 1.25$ & $40 \pm 3.72$ \\
\hline \multirow{2}{*}{ PBW 550} & NS & $39 \pm 1.74$ & $56 \pm 4.63$ & $60 \pm 3.43$ & $37 \pm 2.46$ & $33 \pm 2.83$ \\
\hline & $\mathbf{L S}$ & $35 \pm 1.39$ & $89 \pm 6.34$ & $51 \pm 2.47$ & $28 \pm 1.25$ & $50 \pm 2.54$ \\
\hline \multirow{2}{*}{ Inqalab } & NS & $26 \pm 1.21$ & $54 \pm 2.48$ & $101 \pm 8.49$ & $53 \pm 2.69$ & $42 \pm 1.43$ \\
\hline & $\mathbf{L S}$ & $33 \pm 1.15$ & $109 \pm 8.42$ & $79 \pm 6.21$ & $52 \pm 3.25$ & $43 \pm 3.24$ \\
\hline \multirow{2}{*}{ WL 711} & NS & $29 \pm 1.07$ & $51 \pm 3.71$ & $105 \pm 7.89$ & $55 \pm 3.04$ & $32 \pm 2.68$ \\
\hline & $\mathbf{L S}$ & $39 \pm 2.32$ & $111 \pm 8.56$ & $80 \pm 5.45$ & $48 \pm 2.43$ & $43 \pm 2.43$ \\
\hline \multirow{2}{*}{ Sonalika } & NS & $26 \pm 1.64$ & $48 \pm 3.61$ & $53 \pm 4.61$ & $43 \pm 3.47$ & $29 \pm 1.48$ \\
\hline & LS & $29 \pm 2.25$ & $88 \pm 5.85$ & $42 \pm 2.48$ & $37 \pm 2.32$ & $40 \pm 2.57$ \\
\hline
\end{tabular}

synthesis of cellulose, pectins and starch. An alternative pathway for sucrose hydrolysis in the metabolism of sucrose to starch is via invertases.

Within cereal sink itself, there may be variation in sucrose metabolism e.g. during endosperm development in barley, incoming sucrose is converted to UDP-glucose and fructose by UDP dependent SS (sucrose synthase) pathway [32], whereas in maize endosperms, sucrose may be hydrolyzed initially to free monosaccharides by invertase [33]. Amongst invertases, acid invertase showed a consistently higher activity than neutral invertase throughout grain development in all genotypes of wheat under normal and late sown conditions. Similar results were reported in maize [33] depicting highest invertase activity at 12 DAA with a steady decline thereafter.

Soluble invertases activity was inhibited by both short term and long term heat stress. These results support the hypothesis that the disruption of kernel growth and more particularly endosperm starch biosynthesis in response to heat stress is mainly associated with changes in carbon utilization and partitioning between the different non structural carbohydrates within the endosperm rather than with a limitation in carbon supply to the kernel [7]. High acid invertase activity was found in rapidly growing tissues such as developing roots of carrot [34] or elongating stems of bean [35] in which sucrose content was low or declined rapidly. Thus the function of acid invertase in these tissues is to hydrolyze sucrose under conditions when there is high demand for growth.

Reduction in amylase activity in wheat kernels was reported earlier [36]. PBW 343 had more starch content than PBW 521. At 40 DAA under normal sown conditions PBW 343 had 70 per cent starch content whereas PBW 521 had 63 per cent starch content. Starch content showed continuous increase till 40 DAA in control plants 
Table 6. Total activity of total amylase (nmole reducing sugars formed/min/g FW) in developing grains of different genotypes of wheat under normal and late sown conditions.

\begin{tabular}{|c|c|c|c|c|c|c|}
\hline \multirow{2}{*}{\multicolumn{2}{|c|}{ Genotypes normal/late sown }} & \multicolumn{5}{|c|}{ Activity of total amylase } \\
\hline & & \multicolumn{5}{|c|}{ Days after anthesis (DAA) } \\
\hline & & 7 & 14 & 21 & 28 & 40 \\
\hline \multirow{2}{*}{ C 306} & NS & $76 \pm 4.21$ & $541 \pm 20.69$ & $968 \pm 37.81$ & $1556 \pm 40.23$ & $1081 \pm 52.39$ \\
\hline & $\mathbf{L S}$ & $72 \pm 3.20$ & $515 \pm 18.41$ & $1027 \pm 20.07$ & $993 \pm 32.61$ & $883 \pm 47.21$ \\
\hline \multirow{2}{*}{ PBW 343} & NS & $86 \pm 3.29$ & $556 \pm 28.90$ & $1010 \pm 26.87$ & $1863 \pm 53.70$ & $1065 \pm 73.24$ \\
\hline & $\mathbf{L S}$ & $75 \pm 2.81$ & $527 \pm 22.72$ & $1140 \pm 17.80$ & $1032 \pm 40.91$ & $947 \pm 52.91$ \\
\hline \multirow{2}{*}{ PBW 502} & NS & $73 \pm 5.62$ & $521 \pm 17.61$ & $925 \pm 22.40$ & $1362 \pm 50.58$ & $1018 \pm 61.89$ \\
\hline & LS & $69 \pm 3.50$ & $498 \pm 25.23$ & $974 \pm 25.63$ & $943 \pm 53.01$ & $843 \pm 40.63$ \\
\hline \multirow{2}{*}{ PBW 509} & NS & $58 \pm 3.41$ & $461 \pm 18.47$ & $930 \pm 30.71$ & $1102 \pm 48.36$ & $917 \pm 22.48$ \\
\hline & $\mathbf{L S}$ & $50 \pm 4.63$ & $446 \pm 12.60$ & $952 \pm 19.87$ & $872 \pm 36.41$ & $609 \pm 25.80$ \\
\hline \multirow{2}{*}{ PBW 521} & NS & $53 \pm 2.69$ & $393 \pm 17.80$ & $845 \pm 21.89$ & $1006 \pm 30.81$ & $904 \pm 27.81$ \\
\hline & $\mathbf{L S}$ & $47 \pm 3.24$ & $363 \pm 14.62$ & $921 \pm 24.20$ & $842 \pm 21.76$ & $583 \pm 32.64$ \\
\hline \multirow{2}{*}{ PBW 522} & NS & $68 \pm 4.37$ & $456 \pm 17.81$ & $875 \pm 15.65$ & $1083 \pm 26.12$ & $967 \pm 24.62$ \\
\hline & $\mathbf{L S}$ & $52 \pm 2.52$ & $417 \pm 18.70$ & $949 \pm 19.71$ & $897 \pm 30.62$ & $638 \pm 22.08$ \\
\hline \multirow{2}{*}{ PBW550 } & NS & $74 \pm 4.62$ & $501 \pm 12.29$ & $896 \pm 14.71$ & $1303 \pm 31.63$ & $1005 \pm 18.19$ \\
\hline & $\mathbf{L S}$ & $57 \pm 3.22$ & $482 \pm 11.68$ & $1012 \pm 18.22$ & $968 \pm 25.61$ & $926 \pm 14.60$ \\
\hline \multirow{2}{*}{ Inqalab } & NS & $61 \pm 3.89$ & $571 \pm 20.71$ & $890 \pm 25.80$ & $1080 \pm 37.80$ & $935 \pm 14.67$ \\
\hline & $\mathbf{L S}$ & $52 \pm 2.44$ & $446 \pm 23.40$ & $956 \pm 26.48$ & $904 \pm 28.94$ & $741 \pm 12.72$ \\
\hline \multirow{2}{*}{ WL 711} & NS & $67 \pm 4.73$ & $498 \pm 27.61$ & $1009 \pm 28.93$ & $1267 \pm 24.66$ & $1029 \pm 32.81$ \\
\hline & $\mathbf{L S}$ & $55 \pm 3.14$ & $467 \pm 20.89$ & $1125 \pm 22.08$ & $1007 \pm 19.43$ & $855 \pm 34.60$ \\
\hline \multirow{2}{*}{ Sonalika } & NS & $65 \pm 3.81$ & $462 \pm 19.61$ & $901 \pm 22.19$ & $1105 \pm 20.30$ & $949 \pm 40.63$ \\
\hline & LS & $53 \pm 2.27$ & $429 \pm 25.73$ & $1019 \pm 17.42$ & $916 \pm 18.69$ & $724 \pm 31.78$ \\
\hline
\end{tabular}

in all cultivars [37]. The presence of high activities of starch hydrolyzing enzymes in grain during active starch accumulation period has raised a fundamental question on the specific role played by these enzymes in grain development. In general these enzymes have been implicated either in the breakdown of starch for the production of energy through metabolism of its released glucose monomers and or generation of primer molecules for starch synthetases [38].

It may be possible that increased sugars somehow activate the genes involved in stress tolerance. In addition to role of sucrose as major transported assimilate, there is now compelling evidence, that sucrose is an important mobile signal that regulate a variety of different genes and processes in various plant organs [39]. Starch content was higher in developing grains of heat tolerant genotype i.e. PBW 343 than heat susceptible genotype i.e. PBW 521 at maturity. Premature cessation of starch deposition occurred in both genotypes during late sown conditions which indicated pre advanced maturity of developing grains at high temperature during late sown condition. Decline in free sugars and starch during late sown conditions further indicated that terminal heat stress had caused more utilization of carbohydrates to overcome high temperature stress.

Amongst different genotypes studied PBW 343, C 306 have higher carbon partitioning and grain sink activity while PBW 521, PBW 522 are susceptible and PBW 550, 
PBW 509, Sonalika, WL 711, Inqalab, PBW 502 have been found to be intermediate. Information on carbohydrate partitioning in diverse genotypes could be exploited for generating germplasm that can tolerate high temperature.

\section{Acknowledgements}

Authors are thankful to Department of Biotechnology (DBT), New Delhi, India for providing research grant to carry out this work.

\section{REFERENCES}

[1] H. Zhao, T. Dai, Q. Jing, D. Jiang and W. Cao, "Leaf Senescence and Grain Filling Affects by Post Anthesis High Temperatures in Two Different Wheat Cultivars," Plant Growth Regulation, Vol. 51, No. 2, 2007, pp. 149-158. doi:10.1007/s10725-006-9157-8

[2] G. K. McDonald, B. G. Sutton and F. W. Ellison, "The Effect of Time of Sowing on the Grain Yield of Irrigated Wheat in the Naomi Valley, New South Wales," Australian Journal of Agricultural Research, Vol. 34, No. 3, 1983, pp. 229-240. doi:10.1071/AR9830229

[3] N. B. Singh, Y. P. Singh and V. P. N. Singh, "Variation in Physiological Traits in Promising Wheat Varieties under Late Sown Condition," Indian Journal of Plant Physiology, Vol. 10, No. 2, 2005, pp. 171-175.

[4] A. Blum and B. Sinmena, "Wheat Seed Endosperm Utilization under Heat Stress and Its Relationship to Thermotolerance in Autotrophic Plants," Field Crop Research, Vol. 37, No. 3, 1994, pp. 185-191. doi:10.1016/0378-4290(94)90097-3

[5] B. Asthir, A. S. Basra and S. K. Batta, "Fluoride-Induced Alteration of Carbon and Nitrogen Metabolism in Developing Wheat Grains," Biologia Plantarum, Vol. 41, No. 2, 1998, pp. 287-292. doi:10.1023/A:1001895420374

[6] S. S. Bhullar and C. F. Jenner, "Effects of a Brief Episode of Elevated Temperature on Grain Filling in Winter Wheat Ears Cultured on Solutions of Sucrose," Australian Journal of Plant Physiology, Vol. 13, No. 5, 1986, pp. 617-626. doi:10.1071/PP9860617

[7] N. Cheikh and R. J. Jones, "Heat Stress Effects on Sink Activity of Developing Maize Kernels Grown in Vitro," Plant Physiology, Vol. 95, No. 1, 1995, pp. 59-66. doi:10.1111/j.1399-3054.1995.tb00808.x

[8] M. A. B. Wallwork, S. J. Logue, L .C. MacLeod and C. F. Jenner, "Effects of High Temperature during Grain Filling on Starch Synthesis in the Developing Barley Grain," Australian Journal of Plant Physiology, Vol. 25, No. 2, 1998, pp. 173-181. doi:10.1071/PP97084

[9] L. C. Ho, "Metabolism and Compartmentation of Imported Sugars in Sink Organs in Relation to Sink Strength," Annual Review of Plant Physiology Molecular Biology, Vol. 39, 1988, pp. 355-378.

doi:10.1146/annurev.pp.39.060188.002035

[10] J. Yang, J. Zhang, Z. Wang, G. Xu and Q. Zhu, “Activi- ties of Key Enzymes in Sucrose to Starch Conversion in Wheat Grains Subjected to Water Deficit during Grain Filling," Plant Physiology, Vol. 135, No. 3, 2004, pp. 1621-1629. doi:10.1104/pp.104.041038

[11] C. A. Lovell, P. T. Tomlinson, K. E. Koch, "Sucrose Metabolizing Enzymes in Transport Tissues and Adjacent Sink Structures in Developing Citrus Fruit," Plant Physiology, Vol. 90, No. 4, 1989, pp. 1394-1402. doi:10.1104/pp.90.4.1394

[12] D. P. Xu, S. S. Sung and C. C. Black, "Sucrose Metabolism in Lema Bean Seeds," Plant Physiology, Vol. 89, No. 4, 1989, pp. 1106-1116. doi:10.1104/pp.89.4.1106

[13] L. I Tian, L. Qi-Hua, R. Ohsugi, T. Yamagishi, D. H Sasaki, "Effect of High Temperature on Sucrose Content and Sucrose Cleaving Enzyme Activity in Rice Grain during the Filling Stage," Rice Science, Vol. 13, No. 3, 2006, pp. 205-210.

[14] A. Wahid, S. Gelani, M. Ashraf and M. R. Foolad, "Heat Tolerance in Plants: An Overview," Environmental and Experimental Botany, Vol. 61, No. 3, 2007, pp. 199-223. doi:10.1016/j.envexpbot.2007.05.011

[15] H. T. Buck, J. E. Nisi and N. Salomon (Eds.), "Wheat Production in Stressed Environments," Springer, Amsterdam, 2007, pp. 365-371.

[16] B. Venkateswarlu and R. M. Visperas, "Sucrose-Sink Relationships in Crop Plants," International Rice Research Paper Series, Vol. 125, 1987, pp. 1-19.

[17] J. S. Liang, J. H. Zhang and X. Z. Cao, "Grain Sink Strength May Be Related to the Poor Grain Filling of indica-japonica Rice (Oryza sativa) Hybrids," Plant Physiology, Vol. 112, No. 4, 2001, pp. 470-477. doi:10.1034/j.1399-3054.2001.1120403.x

[18] U. Wobus, N. Sreenivasulu, L. Borisjuk, H. Rolletschek, R. Panitz, S. Gubatz and W. Weschke, "Molecular Physiology and Genomics of Developing Barley Grains," Recent Research Development in Plant Molecular Biology, Vol. 2, 2005, pp. 1-29.

[19] C. F. Jenner, "An Investigation of the Association between the Hydrolysis of Sucrose and Its Absorption by Grains of Wheat," Australian Journal of Plant Physiology, Vol. 1, No. 3, 1974, pp. 319-329. doi:10.1071/PP9740319

[20] B. Asthir, A. S. Basra and S. K. Batta, "Differential Response of Carbon and Nitrogen Metabolism to Fluoride Application in Fruiting Structures of Chickpea," Acta Physiologia Plantarum, Vol. 21, No. 1, 1999, pp. 67-73. doi:10.1007/s11738-999-0029-5

[21] O. H Lowry, N. J Rosebrough, A. L. Farr and R. J. Ranadall, "Protein Measurement with the Folin Phenol Reagent," Journal of Biological Chemistry, Vol. 193, No. 1, 1951, pp. 26-75.

[22] N. Nelson, "A Photometric Adaptation of Somogyi Method for the Determination of Glucose," Journal of Biological Chemistry, Vol. 153, No. 2, 1944, pp. 375-380.

[23] E. Acevedo, M. Nachit and G. Ferrara Ortiz, "Effects of Heat Stress on Wheat and Possible Selection Tools for Use in Breeding for Tolerance," In: D. A. Saunders, Ed., Wheat for the Nontraditional Warm Areas, Centro In- 
ternacional de Mejoramiento de Maiz y Trigo, Mexico City, 1991, pp. 401-421.

[24] F. Rook, S. A Hadingham, Y. Li and M. W. Bevan, "Sugar and ABA Response Pathways and the Control of Gene Expression," Plant Cell and Environment, Vol. 29, No. 3, 2006, pp. 426-434. doi:10.1111/j.1365-3040.2005.01477.x

[25] S. C. B. R. Jose, E. V. R. Von Pinho and M. A. G. S. Dias, "Sugars and Tolerance to High Drying Temperature in Corn Seeds," Revista Brasileira de Sementes, Vol. 28, 2006, pp. 60-68.

[26] E. M. Dale and T. L. Housely, "Sucrose Synthase Activity in Developing Wheat Endosperms Differing in Maximum Weight," Plant Physiology, Vol. 82, No. 1, 1986, pp. 7-10. doi:10.1104/pp.82.1.7

[27] S. Bala, B. Asthir and N. S. Bains, "High Temperature Response Leads to Altered Membrane Permeability in Conjunction with Carbon Utilization in Wheat," Seed Science \& Biotechnology, Vol. 4, No. 1, 2010, pp. 10-14.

[28] Q. Zhou, D. Jiang, T. Dai, Q. Jing and W. Cao, "Regulation of Starch and Protein Synthesis in Wheat Grains by Feeding Sucrose and Glutamine to Detached Ears Cultured in Vitro," Plant Growth Regulation, Vol. 48, No. 3, 2006, pp. 247-259

[29] S. S. Sung, W. J. Sheih, D. R. Geigre and C. C. Black, "Growth, Sucrose Synthase and Invertase Activities of Developing Phaseolus vulgaris L. Fruits," Plant Cell \& Environment, Vol. 17, No. 4, 1994, pp. 419-426. doi:10.1111/j.1365-3040.1994.tb00310.x

[30] S. C. Huber and T. Akazawa, "A Novel Sucrose Synthase Pathway for Sucrose Degradation in Cultured Sycamore Cell," Plant Physiology, Vol. 81, No. 4, 1986, pp. 10081013. doi:10.1104/pp.81.4.1008

[31] G. Avigad and P. M. Dey, "Carbohydrate Metabolism: Storage Carbohydrates," In: P. M. Dey and J. B. Harborne, Eds., Plant Biochemistry, Academic Press, Cam- bridge, 2000, pp. 143-203.

[32] C. M. Duffus and R. Rosie, "Starch Hydrolysing Enzymes in the Developing Barley Grain," Planta, Vol. 109, No. 2, 1973, pp. 153-160. doi:10.1007/BF00386123

[33] C. Y, Tsai, F. Salamini and O. E. Nelson, "Enzymes of Carbohydrate Metabolism in Developing Endosperm of Maize," Plant Physiology, Vol. 4, No. 2, 1970, pp. 299306. doi: $10.1104 /$ pp.46.2.299

[34] C. P. P Ricardo and T. après, "Invertase Activity during the Development of Carrot Roots," Phytochemistry, Vol. 9, No. 2, 1970, pp. 239-247. doi:10.1016/S0031-9422(00)85130-4

[35] D. A. Morris and E. D. Arthur, "Invertase Activity, Carbohydrate Metabolism and Cell Expansion in the Stem of Phaseolus vulgaris L.," Journal of Experimental Botany, Vol. 36, No. 4, 1985, pp. 623-633. doi: $10.1093 / \mathrm{jxb} / 36.4 .623$

[36] S. Alka and R. Khanna-Chopra, "Influence of Temperature on Germination and Seedling Growth and Its Relationship with Amylase Activity and Respiration in Wheat Varieties Differing in Temperature Tolerance," Indian Journal of Experimental Biololgy, Vol. 33, No. 11, 1995, pp.775-779.

[37] P. Prakash, P. Sharma-Natu and M. C. Ghildiyal, "High Temperature Effect on Starch Synthase Activity in Relation to Grain Growth in Wheat Cultivars," Indian Journal of Plant Physiology, Vol. 8, 2003, pp. 390-398.

[38] C. M. Duffus and M. P. Cochrane, "Grain Structure and Composition,” In: P. R. Shewry Ed., Barley: Genetics, Molecular Biology and Technology, C.A.B. International, London, 1992, pp. 219-317.

[39] J. Yang, J. Zhang, Z. Wang, G. Xu and Q. Zhu, "Activities of Key Enzymes in Sucrose to Starch Conversion in Wheat Grains Subjected to Water Deficit during Grain Filling," Plant Physiology, Vol. 135, No. 3, 2004, pp. 1621-1629. doi:10.1104/pp.104.041038 